\title{
TDP-43 protein in plasma may index TDP-43 brain pathology in Alzheimer's disease and frontotemporal lobar degeneration
}

\author{
Penelope Foulds · Erica McAuley • Linda Gibbons • Yvonne Davidson • \\ Stuart M. Pickering-Brown · David Neary · Julie S. Snowden · \\ David Allsop · David M. A. Mann
}

Received: 16 April 2008 / Revised: 8 May 2008 / Accepted: 8 May 2008 / Published online: 28 May 2008

(C) The Author(s) 2008

\begin{abstract}
Autopsy studies have shown that about 55\% of patients with frontotemporal lobar degeneration (FTLD) and $25 \%$ of patients with Alzheimer's disease (AD) harbour TDP-43 immunoreactive pathological changes in their brains. Using ELISA, we investigated whether we could detect the presence, or increased amounts, of TDP-43 in plasma of patients with FTLD and AD compared to normal control subjects. We detected elevated levels of TDP-43 protein in plasma of $46 \%$ patients with FTLD with clinical frontotemporal dementia (FTD) and $22 \%$ patients with $\mathrm{AD}$, compared to $8 \%$ of control subjects. The proportions of patients with FTD and AD showing raised plasma TDP-43 levels correspond closely to those proportions known from autopsy studies to contain TDP-43 pathological changes in their brains. Raised TDP-43 plasma levels may thereby
\end{abstract}

P. Foulds and E. McAuley contributed equally to the study.

P. Foulds $\cdot$ E. McAuley $\cdot$ D. Allsop

Division of Biomedical and Life Sciences,

School of Health and Medicine,

University of Lancaster, Lancaster, UK

L. Gibbons · Y. Davidson · D. Neary · J. S. Snowden ·

D. M. A. Mann ( $\square)$

Clinical Neurosciences Research Group,

School of Translational Medicine,

Faculty of Medical and Human Sciences,

University of Manchester, Greater Manchester

Neurosciences Centre, Hope Hospital,

Salford M6 8HD, UK

e-mail: david.mann@manchester.ac.uk

S. M. Pickering-Brown

Clinical Neurosciences Research Group,

School of Translational Medicine,

Faculty of Medical and Human Sciences,

University of Manchester, Oxford Road,

Manchester M13 9PT, UK index TDP-43 pathology within the brain. Plasma TDP-43 levels may be a biomarker that can provide a laboratory test capable of identifying the presence of TDP-43 brain pathology in neurodegenerative disease during life. It may help to distinguish those cases of FTLD with ubiquitin/TDP-43 pathology in their brains from those with tauopathy. As a predictive test, plasma TDP-43 level may have great practical value in directing therapeutic strategies aimed at preventing or removing tau or TDP-43 pathological changes from the brain in FTLD and AD.

Keywords Frontotemporal lobar degeneration . Alzheimer's disease $\cdot$ TDP-43 $\cdot$ Plasma $\cdot$ Biomarker

\section{Introduction}

Recent research in neurodegenerative disease has highlighted the importance of a new molecular entity, the TAR DNA binding protein, TDP-43 (see [6] for review). Prior to the discovery in 2006 that TDP-43 was the target protein in the ubiquitinated cytoplasmic inclusions in neurones in certain histological forms of frontotemporal lobar degeneration (FTLD) known as FTLD-ubiquitinated (FTLD-U), and in motor neurone disease (MND) [2, 24], little was known of the function of this 414 amino acid nuclear protein beyond it serving a role in nuclear transcription in relationship to alternative splicing or exon skipping $[5,30]$ The involvement of TDP-43 in the ubiquitinated lesions of FTLD-U and MND has now been amply confirmed [6, 7, 9, 17, 29]. While ubiquitinated TDP-43 lesions are characteristically seen in those patients with inherited forms of FTLD associated with mutations in progranulin gene $(P G R N)[3,4,8,9,16,26,28]$, in other patients with FTLD, such as those with mutations in tau gene (MAPT), or 
in those with a Pick body-type histology, where the underlying histology is based on the accumulation of aggregated tau proteins, no such TDP-43 pathological changes are seen [9]. Moreover, other studies have shown that TDP-43 pathological changes can also occur in about $20 \%$ of patients with Alzheimer's disease (AD) [1] and are widely present in patients with Lewy body diseases [21] or with parkinsonism-dementia complex of Guam [12].

Present data therefore suggest a widespread involvement of TDP-43 in neurodegenerative disease beyond FTLD-U and MND. In clinical terms, about $70 \%$ of patients with FTLD show behavioural and personality changes which are collectively described as frontotemporal dementia (FTD) [23]. Prospective and retrospective pathological studies have indicated that about half of all cases with FTD have a tau-positive, TDP-43-negative pathology, and half have ubiquitin-positive, TDP-43-positive pathology [10, 14, 15, 20,27]. Nevertheless, in patients with FTLD, it is presently not possible to predict, in life, what the underlying histological changes might be solely on the basis of clinical phenotype, and it is only in that minority of patients where mutations in MAPT or PGRN are present that a tau or ubiquitin/TDP-43 based histology, respectively, can be confidently inferred.

The differential diagnosis of dementia syndromes is clinical and radiological, and there are no litmus tests based on measures in blood or plasma to specify diagnosis. A biochemical marker that could distinguish between $\mathrm{AD}$ and FTLD, or between the histological subtypes of FTLD, would be not only of great diagnostic value, but would be highly pertinent to the future development of drugs aimed at preventing or removing the intraneuronal accumulation of tau or TDP-43 proteins in these degenerative disorders. Given the pathological observations that a proportion of patients with AD [1] or FTLD [9, 14, 15, 19, 27] harbour TDP-43 pathological changes within their brains, it can be hypothesised that similar proportions of patients might show altered blood or plasma levels of TDP-43, and that this could be a useful biomarker of the presence of TDP-43 brain pathology.

In this present study we have investigated whether TDP43 can be detected in plasma in patients with FTD and AD, and in normal control subjects, by developing an ELISAbased assay. We show that not only can TDP-43 be detected in plasma, but more importantly, that the level of this is elevated in a proportion of each group of patients similar to that seen in patients with known TDP-43 brain pathology. Measurement of plasma TDP-43 may therefore have utility in evidencing the presence of brain TDP-43 based pathologies in neurodegenerative disease, and perhaps more importantly be capable of discriminating during life those patients with FTLD associated with ubiquitin/ TDP-43 pathological changes in their brains from those with tauopathy, when a defining mutation in MAPT or $P G R N$ is absent.

\section{Materials and methods}

Blood samples

Blood samples were obtained with Ethical permissions from 137 patients, 35 with FTD and 102 with $\mathrm{AD}$, and 85 nondemented control subjects (Table 1). All patients with FTD and AD had been assessed (by DN, JSS) within out-patient clinics at the Cerebral Function Unit, Greater Manchester Neurosciences Centre. All patients had undergone historical interview in the presence of a family caregiver and comprehensive cognitive assessment to obtain information about the nature and progression of cognitive and functional decline. Patients with AD and FTD fulfilled the National Institute of Neurological and Communicative Disorders and Stroke-Alzheimer's Disease and Related Disorders Association (NINCDS-ADRDA) criteria for probable AD [18] and International criteria for FTD [22], respectively. Control subjects were either spouses of patients attending CFU clinic $(n=13)$, or a sample of healthy volunteers $(n=72)$ drawn randomly from a cohort of 767 mentally normal people aged $>50$ years, resident within the same Greater Manchester region from which the AD and FTD patients were recruited [25]. The latter comprised a longitudinal ageing study group who had been screened psychologically for absence of cognitive impairment consistent with dementia. All blood samples had been collected with the approval of the local ethics research committee.

Plasma was separated from whole blood samples $(5 \mathrm{ml}$ blood with EDTA acting as anti-coagulant) by routine methods, and stored in deep freeze $\left(-80^{\circ} \mathrm{C}\right)$ until assay.

Table 1 Details of groups of patients with AD and FTD, and control subjects, at the time when blood samples were taken

\begin{tabular}{|c|c|c|c|}
\hline & Control & $\mathrm{AD}$ & FTD \\
\hline Patients $(n)$ & 85 & 102 & 35 \\
\hline Age range (years) & $42-83$ & $43-88$ & $42-78$ \\
\hline Mean age (years) \pm SD & $71.3 \pm 8.3$ & $64.2 \pm 9.3$ & $62.4 \pm 8.2$ \\
\hline $\begin{array}{l}\text { Mean age of onset } \\
\quad \text { (years) } \pm S D\end{array}$ & $\mathrm{n} / \mathrm{a}$ & $61.5 \pm 9.4$ & $69.7 \pm 8.4$ \\
\hline $\begin{array}{l}\text { Mean disease duration } \\
\quad \text { (years) } \pm \mathrm{SD}\end{array}$ & $\mathrm{n} / \mathrm{a}$ & $2.9 \pm 1.8$ & $2.7 \pm 2.1$ \\
\hline Gender: Male:Female\% $(n)$ & $\begin{array}{r}43.5: 56.5 \\
(37: 48)\end{array}$ & $\begin{array}{r}51.9: 48.1 \\
(53: 49)\end{array}$ & $\begin{array}{r}48.6: 51.4 \\
\quad(17: 18)\end{array}$ \\
\hline Mean absorbance value & 0.02 & 0.13 & 0.26 \\
\hline Median absorbance value & -0.04 & 0.02 & 0.11 \\
\hline $\begin{array}{l}\text { Percentage of samples over } \\
\text { the } 0.11 \text { cut-off point }(\%)\end{array}$ & 8.8 & 23 & 46 \\
\hline
\end{tabular}


Immunoassay protocol

An antibody-sandwich ELISA was developed to measure the TDP-43 concentration in plasma samples. The ELISA plates (96-well PVC assay plates, Iwaki, Japan) were coated by overnight incubation at $4^{\circ} \mathrm{C}$ with $0.2 \mu \mathrm{g} / \mathrm{ml}$ antiTDP monoclonal antibody (H00023435-M01, clone 2E2D3, Abnova Corporation, Taiwan), $100 \mu \mathrm{l} /$ well, diluted in $200 \mathrm{mM} \mathrm{NaHCO} 3$ buffer, pH 9.6, containing 0.02\% (w/v) sodium azide. The plates were washed 3 times with PBST (PBS (0.01 M phosphate buffer, 0.0027 M potassium chloride and $0.137 \mathrm{M}$ sodium chloride, $\mathrm{pH}$ 7.4) containing $0.05 \%$ Tween 20), and incubated with $200 \mu \mathrm{l} /$ well of blocking buffer (PBS containing $2.5 \%$ gelatin and $0.05 \%$ Tween $20)$ for $2 \mathrm{~h}$ at $37^{\circ} \mathrm{C}$. The plates were again washed 3 times with PBST, and $100 \mu$ of the plasma samples to be tested, diluted 1:1 with PBS, were added to each of three wells. After washing 3 times with PBST, $100 \mu$ of the detection antibody, TDP rabbit polyclonal antibody (BC001487, ProteinTech Group, Chicago) diluted to $0.2 \mu \mathrm{g} / \mathrm{ml}$ in blocking buffer, was added per well and the plates were incubated at $37^{\circ} \mathrm{C}$ for $2 \mathrm{~h}$. After another wash (as before), the plates were incubated with $100 \mu \mathrm{l} /$ well of goat anti-rabbit secondary antibody coupled to horseradish peroxidase (HRP) (Dako Ltd., Ely, U.K.), diluted 1:10,000 in blocking buffer, at $37^{\circ} \mathrm{C}$ for $1 \mathrm{~h}$. The plates were then washed again with PBST, before adding $100 \mu \mathrm{l} /$ well Sure Blue TMB Microwell Peroxidase Substrate (KPL Inc, Maryland, USA) and leaving the colour to develop for $30 \mathrm{~min}$ at room temperature. Finally, $100 \mu \mathrm{l} /$ well of stop solution $\left(0.3 \mathrm{M} \mathrm{H}_{2} \mathrm{SO}_{4}\right)$ was added and absorbance values were read at $450 \mathrm{~nm}$ in a Victor $^{2}$ multi-function microtitre plate reader. Net absorbance was calculated by deducting the mean value obtained for a triplicate of "blank" wells containing PBS only.

The antibody-sandwich ELISA using polyclonal TDP43 antibody (BC001487) as capture antibody was also successful and gave similar signals. However, the monoclonal antibody (2E2-D3) was chosen as the preferred capture antibody as background signals were smaller.

Recombinant TDP-43 protein (MW 54.3 KDa) (AAH01487, 1 -261, Abnova Corporation, Taiwan, with 26 KDa GST tag) was used in the standard curve.

Immunoprecipitation of TDP-43 from plasma

Dynabeads covalently coupled with recombinant protein A, were derivatised with rabbit polyclonal anti-TDP-43 antibody (BC001487, ProteinTech Group, Chicago) as recommended by the manufacturer (Dynal Biotech LTD.,Wirral, UK). $300 \mu \mathrm{l}$ plasma was added to the beads and incubated overnight at $4{ }^{\circ} \mathrm{C}$. The plasma samples were chosen according to the ELISA results, with one sample giving a high signal and the other a low signal. The beads were then washed three times with $0.1 \mathrm{M}$ phosphate buffer, $\mathrm{pH}$ 8.2. Any captured TDP-43 was eluted from the beads by boiling for 10 min in NuPAGE LDS sample buffer (Invitrogen Ltd., UK) and examined by gel electrophoresis and immunoblotting.

\section{Gel electrophoresis and immunoblotting}

The protein eluted from the magnetic dynabeads (see above) was separated on NUPAGE Bis-Tris 4-12\%, $1 \mathrm{~mm}$ gels (Invitrogen). The separated proteins were transferred to nitrocellulose membranes $(0.45 \mu \mathrm{m}$, Invitrogen $)$ at $30 \mathrm{~V}$, $125 \mathrm{~mA}$ for $1 \mathrm{~h}$. Membranes were blocked with 5\% Marvel dried skimmed milk, dissolved in PBST for $1 \mathrm{~h}$. The membranes were probed overnight at $4^{\circ} \mathrm{C}$ with either anti-TDP43 antibody, rabbit polyclonal (BC001487, ProteinTech Group, Chicago) 1:1,000 in PBST, or anti-TDP-43 mouse monoclonal antibody (H00023435-M01, clone 2E2-D3, Abnova Corporation, Taiwan) 1:1,000 in PBST, as indicated. The membranes were washed three times in PBST, followed by incubation with HRP-conjugated goat anti-rabbit or goat anti-mouse (Dako LTD., Ely, U.K.), 1:10,000 in PBST, as appropriate, for $1 \mathrm{~h}$. The protein bands were visualised using ECL reagents (Pierce, Rockford, IL) as described by the manufacturer.

\section{Statistical analyses}

All data were analysed using SPSS v 14.0. Because OD values for FTD and AD patients, as well as control subjects were not normally distributed according to KolmogorovSmirnov test, non-parametric Kruskal-Wallis test was used throughout to compare groups, with post hoc Mann-Whitney test being employed when the results of Kruskal-Wallis test yielded significant group differences.

\section{Results}

A standard curve for the ELISA is shown in Fig. 1. The ELISA data from the plasma samples indicate that in control subjects, in most instances, TDP-43 was barely detectable (mean $\pm \mathrm{SD}, \mathrm{OD}=0.02 \pm 0.32$ ), with just 8 individuals $(8.8 \%)$ having absorbance (optical density or OD) values exceeding 0.11 (Fig. 2). This value corresponds to the $99 \%$ upper confidence level and was subsequently chosen to represent the 'cut off' point of 'normal' TDP-43 values. In AD, TDP-43 was again barely detectable in 79 patients $(78 \%)$ (Fig. 2), though OD exceeded 0.11 in 23 patients (22\%). In FTD, $16 / 35$ patients (46\%) patients had OD value exceeding 0.11 (Fig. 2). The proportion of patients with OD greater than 0.11 differed significantly across the groups $\left(\chi^{2}=19.8\right.$; $P<0.001)$ with the proportion of patients with OD values greater than 0.11 being significantly higher in both FTD 


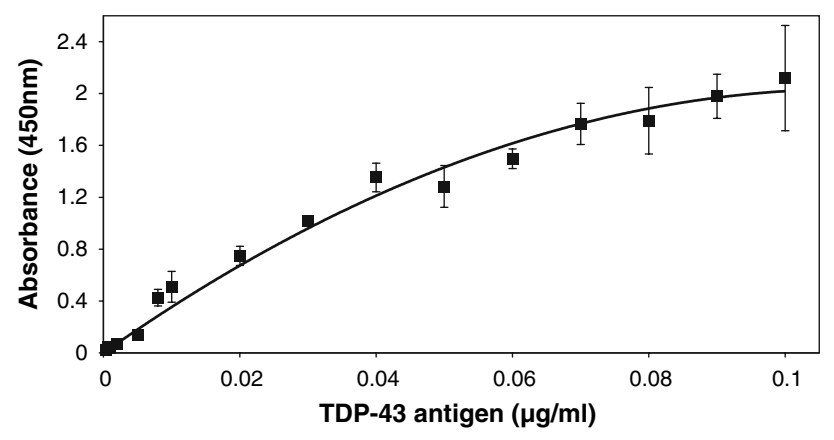

Fig. 1 Standard curve for the TDP-43 ELISA (data show mean \pm SD)

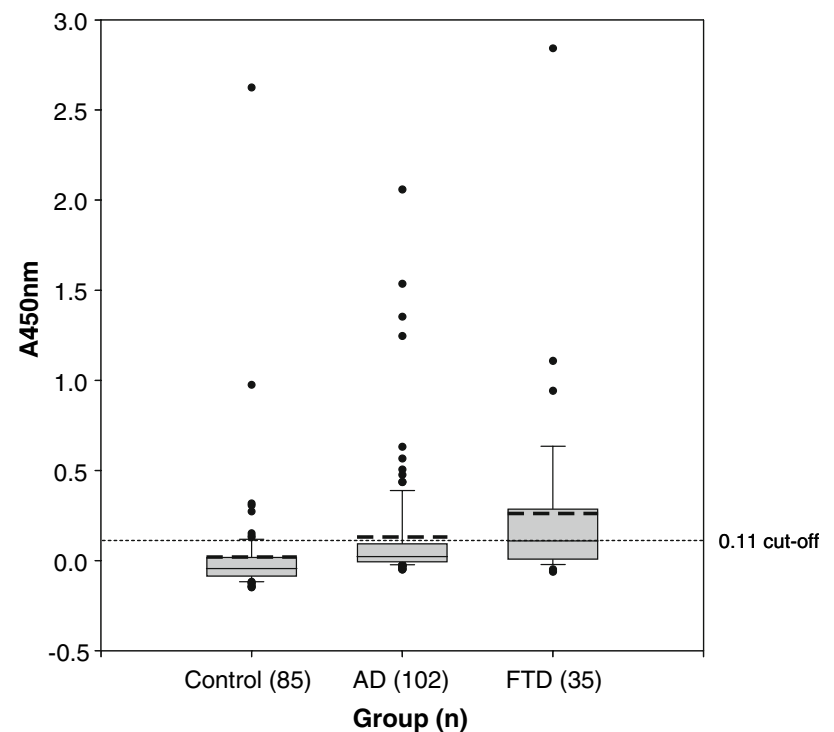

Fig. 2 Box-whisker plots for the plasma ELISA results (absorbance values). The length of each box represents the interquartile range (75$25 \%$ ) of the sample, the solid line drawn across the box the median, the dashed line the mean, and outliers are denoted by dots. The dotted line representing the 0.11 cut-off corresponds to the $99 \%$ upper confidence level for the control group

$\left(\chi^{2}=20.4 ; P<0.001\right)$ and $\mathrm{AD}\left(\chi^{2}=5.8 ; P=0.016\right)$ compared to controls, and in FTD compared to $\mathrm{AD}\left(\chi^{2}=6.9\right.$; $P=0.009)$. Consequently, the mean OD values for control subjects $(0.02 \pm 0.32)$ and patients with FTD $(0.26 \pm 0.52)$ and $\mathrm{AD}(0.13 \pm 0.33)$ were significantly different by KruskalWallis test $\left(\chi^{2}=53.2 ; P<0.001\right)$, with post hoc MannWhitney test showing mean values for both FTD and AD groups to be significantly different from controls $(P<0.001)$, with mean OD in FTD group also being significantly greater $(P=0.037)$ than that in AD.

When protein from plasma samples with high levels of TDP-43 (based on ELISA results) was immunocaptured with magnetic Dynabeads coupled to the anti-TDP-43 rabbit polyclonal antibody, and then examined by gel electrophoresis and western blotting employing the anti-TDP-43 monoclonal antibody for detection, only a single band migrating at $\sim 43 \mathrm{kDa}$ was seen (Fig. 3, lane 2). No specific
Fig. 3 Immunoblotting results. Lane 1 molecular weight markers (numbers to left in $\mathrm{kDa}$ ); lane 2 protein immunocaptured from a plasma sample with a high ELISA reading

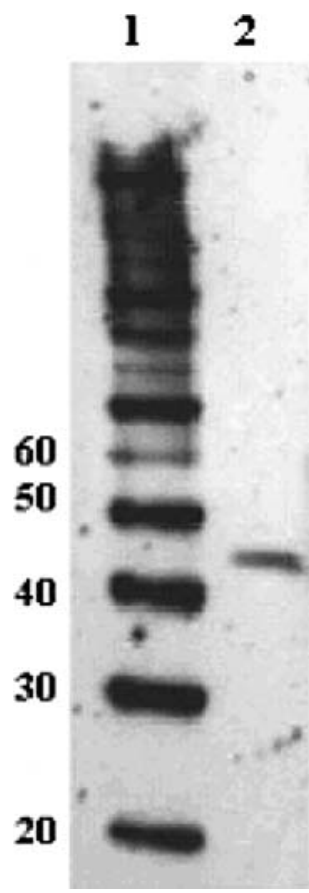

bands were detected in similarly treated low-reading plasma samples.

There were no significant correlations between plasma TDP-43 level and age at onset of illness, or duration of illness up to time of sampling, for AD and FTD groups, either when the whole AD or FTD group, or just those individuals with high TDP-43 levels, were considered.

\section{Discussion}

In the present study we have shown for the first time that the TAR DNA-binding protein, TDP-43, can be detected within plasma of normal control people, as well as individuals with neurodegenerative diseases such as AD and FTD. The ELISA data indicate that TDP-43 is present at extremely low concentrations, or is absent, in the vast majority of normal people, and in AD or FTD there are substantial numbers of patients in whom TDP-43 is likewise barely present. However, group comparisons by ELISA reveal a highly significant increase (approximately 13-fold) in mean TDP-43 absorbance levels in FTD compared to controls, whereas in AD the mean TDP-43 absorbance level was about 7-fold increased. The validity of the ELISA method is supported by the immunoblotting results. In accord with the ELISA data, an immunoreactive band migrating at $43 \mathrm{kDa}$, which corresponds precisely to the molecular weight of full-length TDP-43 [2, 24] was detected only in the high-reading plasma sample and not in the low-reading sample. This result not only confirms the specificity of the antibody pair employed for the sandwich 
ELISA, but also shows that the readout from the ELISA accurately reflects the levels of TDP-43 present in plasma.

From histological studies, it is known that about $50 \%$ of patients with FTD have ubiquitin/TDP-43-based histology $[6,7,10,14,15,20,27]$ and consequently it might be expected that a similar proportion of patients with this kind of pathology would be included within the present unselected FTD study group. In the present FTD cohort, there was a highly significant mean increase in plasma TDP-43 absorbance levels, with about $46 \%$ patients showing 'high' TDP-43 levels (ie above OD of 0.11 corresponding to $99 \%$ upper control limit). On the basis of autopsy studies [6, 7, 9] such a proportion of patients would have been anticipated if the presence of TDP-43 in plasma is indeed indexing the presence of TDP-43 pathology in brain. Likewise, in $\mathrm{AD}$ it is known that about $20-25 \%$ patients have TDP43-based pathology [1] and again it might be anticipated that within the present group of unselected AD cases, a similar proportion of cases with TDP-43 pathology would exist. In the present AD group $22 \%$ patients showed 'high' TDP-43 values, while in most patients no increase was seen. Therefore the proportion of patients showing high plasma TDP-43 levels in both FTD and AD closely match, at least in numerical terms, those proportions that would be predicted to occur from histological studies.

The few elderly control subjects with high TDP-43 levels $(8 \%)$ may be patients with incipient neurodegenerative disease, most likely $\mathrm{AD}$, since it has long been known that a very high proportion of mentally normal elderly subjects show at autopsy some degree of Alzheimer-type pathological changes in their brains (ie senile plaques and neurofibrillary tangles), and this may, as in AD itself, include some individuals with additional TDP-43 pathology. Indeed, the proportion of control subjects showing raised plasma TDP-43 levels recorded here is similar to the prevalence rate of elderly individuals with mild cognitive impairment. [11, 13, 19], supporting the view that such individuals might represent cases of early or incipient AD with additional TDP-43 pathological changes.

Although the numerical similarities between the proportions of patients with FTD and AD showing 'high' TDP-43 plasma levels and those displaying TDP-43 pathological changes at autopsy argue that measurement of plasma TDP43 level can index the presence of TDP-43 pathology within the brain of patients with FTD or AD, we accept that these numerical associations may simply be fortuitous, or may reflect, for example, a compromised blood-brain barrier in FTD and AD, and recognise that the data we have presented here, although intriguing, is still very much preliminary. Present data, and the arguments we have presented based on this data, would be greatly strengthened if autopsy follow-up of patients with AD or FTD with high plasma TDP-43 values were available. However, because the patients investigated here were still in the early or middle stages of their illness when blood samples were taken, this will not be possible for many years to come. An alternative approach would be to compare plasma TDP-43 levels in FTD patients bearing MAPT or PGRN mutations, since it is known from histological studies that patients with MAPT mutations and tauopathy do not show TDP-43 pathological changes in their brains [9], whereas those with PGRN mutation always display TDP-43 pathological changes $[3,4,8,9,16,26,28]$. Hence, by the present argument, patients with MAPT mutations should show low (normal) plasma TDP-43 levels and those with PGRN mutations should show high(er) plasma TDP-43 levels. Unfortunately, within the present cohort of FTD patients there was only one bearer of PGRN mutation (in whom the plasma TDP-43 level was raised), and no bearers of MAPT mutation, so at present we are not realistically able to address this issue. We are presently aiming to collect further plasma samples from the surviving MAPT and PGRN mutation bearers within our FTD cohort. Until then, present data suggest, but do not definitively show, that measurement of plasma TDP-43 level might discriminate in life, those patients with FTD or AD with TDP-43 pathology from those without TDP-43 pathology (ie those with taubased histological changes), even if it does not differentiate between patients with AD or FTD per se.

Because all patients investigated were (when sampled) in early to middle stages of disease it has not been possible so far to follow or deduce how changes in plasma TDP-43 levels might change with disease progression or disease severity. Further studies based on repeat sampling from patients with elevated plasma TDP-43 levels will address this matter.

Although we do not know the biological or functional significance of (the additional) TDP-43 pathology in patients with $\mathrm{AD}$, as with FTD, there may be heuristic (future) management or therapeutic values in being able to predict the presence of TDP-43 changes in such patients. Given the possibility of tau- or TDP-43-based therapies becoming available for patients with AD or FTD, knowing who in AD or FTD is potentially able to benefit from tau and/or TDP-43 based therapy, according to predictive knowledge of underlying histology, would be of considerable practical value.

Acknowledgments Penelope Foulds was supported by a Studentship from Lancaster University. Dr. Pickering-Brown is the recipient of a Medical Research Council (MRC) New Investigator Award, and he and Professor Mann receive other funding from MRC, Alzheimer's Research Trust and the Motor Neurone Disease Association.

Open Access This article is distributed under the terms of the Creative Commons Attribution Noncommercial License which permits any noncommercial use, distribution, and reproduction in any medium, provided the original author(s) and source are credited. 


\section{References}

1. Amador-Ortiz C, Lin W-L, Ahmed Z, Personett D, Davies P, Duara R et al (2007) TDP-43 immunoreactivity in hippocampal sclerosis and Alzheimer's disease. Ann Neurol 61:435-445. doi:10.1002/ana.21154

2. Arai T, Hasegawa M, Akiyama H, Ikeda K, Nonaka T, Mori H et al (2006) TDP-43 is a component of ubiquitin-positive tau-negative inclusions in frontotemporal lobar degeneration and amyotrophic lateral sclerosis. Biochem Biophys Res Commun 351:602611. doi:10.1016/j.bbrc.2006.10.093

3. Baker M, Mackenzie IRA, Pickering-Brown SM, Gass J, Rademakers R, Lindholm C et al (2006) Mutations in Progranulin cause tau-negative frontotemporal dementia linked to chromosome 17. Nature 442:916-919. doi:10.1038/nature05016

4. Boeve BF, Baker M, Dickson DW, Parisi JE, Giannini C, Jsephs KA et al (2006) Frontotemporal dementia and parkinsonism associated with the IVS1 $+1 \mathrm{G} \rightarrow$ A mutation in progranulin: a clinicopathologic study. Brain 129:3103-3114. doi:10.1093/brain/ awl268

5. Buratti E, Brindisi A, Pagini F, Baralle FE (2004) Nuclear factor TDP-43 binds to the polymorphic TG repeats in CFTR intron 8 and causes skipping of exon 9: a functional link with disease penetrance. Am J Hum Genet 74:1322-1325. doi:10.1086/420978

6. Cairns NJ, Bigio EH, Mackenzie IRA, Neumann M, Lee VM-Y, Hatanpaa KJ et al (2007) Neuropathologic diagnostic and nosologic criteria for frontotemporal lobar degeneration: consensus of the Consortium for Frontotemporal Lobar Degeneration. Acta Neuropathol 114:5-22. doi:10.1007/s00401-007-0237-2

7. Cairns NJ, Neumann M, Bigio EH, Holm IE, Troost D, Hatenpaa $\mathrm{KJ}$ et al (2007) TDP-43 in familial and sporadic frontotemporal lobar degeneration with ubiquitin inclusions. Am J Pathol 171:227-240. doi:10.2353/ajpath.2007.070182

8. Cruts M, Gijselinck I, van der Zee J, Engelborghs S, Wils H, Pirici $\mathrm{D}$ et al (2006) Null mutations in progranulin cause ubiquitin-positive frontotemporal dementia linked to chromosome $17 \mathrm{q} 21$. Nature 442:920-924. doi:10.1038/nature05017

9. Davidson Y, Kelley T, Mackenzie IR, Du Plessis D, Neary D, Snowden JS et al (2007) Ubiquitinated pathological lesions in frontotemporal lobar degeneration contain the TAR DNA-binding protein, TDP-43. Acta Neuropathol 113:521-533. doi:10.1007/ s00401-006-0189-y

10. Forman MS, Farmer J, Johnson JK, Clark CM, Arnold SE, Coslett $\mathrm{HB}$ et al (2006) Frontotemporal dementia: clinicopathological correlations. Ann Neurol 59:952-962. doi:10.1002/ana.20873

11. Ganguli M, Dodge HH, Shen C, DeKosky ST (2004) Mild cognitive impairment, amnestic type: an epidemiologic study. Neurology 63:115-121

12. Hasegawa M, Arai T, Akiyama H, Nonaka T, Mori H, Hashimoto $\mathrm{T}$ et al (2007) TDP-43 is deposited in the Guam parkinsonismdementia complex brains. Brain 130:1386-1394. doi:10.1093/ brain/awm065

13. Honinen T, Hallikainen M, Tuomainen S, Vanhanen M, Soininen $H$ (2002) Prevalence of mild cognitive impairment: a populationbased study in elderly subjects. Acta Neurol Scand 106:148-154. doi:10.1034/j.1600-0404.2002.01225.x

14. Lipton AM, White CL III, Bigio EH (2005) Frontotemporal lobar degeneration with motor neuron disease-type inclusions predominates in 76 cases of frontotemporal degeneration. Acta Neuropathol 108:379-385. doi:10.1007/s00401-004-0900-9

15. Mackenzie IRA, Baborie A, Pickering-Brown SM, Du Plessis D, Jaros E, Perry RH et al (2006) Heterogeneity of ubiquitin pathology in frontotemporal lobar degeneration. Acta Neuropathol 112:539 549. doi:10.1007/s00401-006-0138-9

16. Mackenzie IRA, Baker M, Pickering-Brown S, Hsiung G-YR, Lindholm C, Dwosh E et al (2006) The neuropathology of frontotemporal lobar degeneration caused by mutations in the progranulin gene. Brain 129:3081-3090. doi:10.1093/brain/awl271

17. Mackenzie IRA, Bigio EH, Ince PG, Geser F, Neumann M, Cairns NJ et al (2007) Pathological TDP-43 distinguishes sporadic amyotrophic lateral sclerosis from Amyotrophic lateral sclerosis with SOD1 mutations. Ann Neurol 61:427-434. doi:10.1002/ana.21147

18. McKhann G, Drachman D, Folstein M (1984) Clinical diagnosis of Alzheimer's disease. Report of the NINCDS-ADRDA Work Group under the auspices of Department of Health and Human Services Task Force on Alzheimer's disease. Neurology 34:939944

19. Meguro K, Ishii H, Yamaguchi S, Ishizaki J, Sato M, Hashimoto $R$ et al (2004) Prevalence and cognitive performances of clinical dementia rating 0.5 and mild cognitive impairment in Japan. The Tajiri project. Alzheimer Dis Assoc Disord 18:3-10. doi:10.1097/ 00002093-200401000-00002

20. Mott RT, Dickson DW, Trojanowski JQ, Zhukareva V, Lee VM, Forman M et al (2005) Neuropathologic, biochemical, and molecular characterization of the frontotemporal dementias. J Neuropathol Exp Neurol 64:420-428

21. Nakashima-Yasuda H, Uryu K, Robinson J, Xie SX, Hurtig H, Duda JE et al (2007) Co-morbidity of TDP-43 proteinopathy in Lewy body related diseases. Acta Neuropathol 114:221-229. doi:10.1007/s00401-007-0261-2

22. Neary D, Snowden JS, Gustafson L, Passant U, Stuss D, Black S et al (1998) Frontotemporal lobar degeneration: A consensus on clinical diagnostic criteria. Neurology 51:1546-1554

23. Neary D, Snowden JS, Mann DMA (2005) Frontotemporal dementia. Lancet Neurol 4:771-779. doi:10.1016/S14744422(05)70223-4

24. Neumann M, Sampathu DM, Kwong LK, Truax AC, Micsenyi MC, Chou TT et al (2006) Ubiquitinated TDP-43 in frontotemporal lobar degeneration and amyotrophic lateral sclerosis. Science 314:130-133. doi:10.1126/science.1134108

25. Payton A, Holland F, Diggle P, Rabbitt P, Horan MA, Davidson $Y$ et al (2003) Cathepsin D exon 2 polymorphism associated with general intelligence in a healthy older population. Mol Psychiatry 8:14-18. doi:10.1038/sj.mp. 4001239

26. Pickering-Brown SM, Baker M, Gass J, Boeve BF, Loy CT, Brooks WS et al (2006) Mutations in progranulin explain atypical phenotypes with variants in MAPT. Brain 129:3124-3126. doi:10.1093/brain/awl289

27. Shi J, Shaw CL, Richardson AMT, Bailey K, Tian J, Varma AR et al (2005) Histopathological changes underlying frontotemporal lobar degeneration with clinicopathological correlation. Acta Neuropathol 110:501-512. doi:10.1007/s00401-005-1079-4

28. Snowden JS, Pickering-Brown SM, Mackenzie IR, Richardson AMT, Varma A, Neary D et al (2006) Progranulin gene mutations associated with frontotemporal dementia and progressive aphasia. Brain 129:3091-3102. doi:10.1093/brain/awl267

29. Tan C-F, Eguchi H, Tagawa A, Onodera O, Iwasaki T, Tsujino A et al (2007) TDP-43 immunoreactivity in neuronal inclusions in familial amyotrophic lateral sclerosis with or without SOD1 gene mutation. Acta Neuropathol 113:535-542. doi:10.1007/s00401007-0206-9

30. Wang HY, Wang IF, Bose J, Shen CK (2004) Structural diversity and functional implications of the eukaryotic TDP gene family. Genomics 83:130-139. doi:10.1016/S0888-7543(03)00214-3 